\title{
The Reality Plan and the Subjective Construction of One's Perception: The Strategic Theoretical Model among Sensations, Perceptions, Defence Mechanisms, Needs, Personal Constructs, Beliefs System, Social Influences and Systematic Errors
}

\section{Giulio Perrotta}

Department of Criminal and Investigative Psychology Studies, University of Federiciana, Cosenza, Italy

Corresponding author: Giulio Perrotta, Department of Criminal and Investigative Psychology Studies, University of Federiciana, Cosenza, Italy. Email: giuliosr1984@hotmail.it

Received date: November 16, 2019; Accepted date: December 02, 2019; published date: December 10, 2019

Citation: Giulio Perrotta. The Reality Plan and the Subjective Construction of One's Perception: The Strategic Theoretical Model among Sensations, Perceptions, Defence Mechanisms, Needs, Personal Constructs, Beliefs System, Social Influences and Systematic Errors. J Clinical Research and Reports, 1(1); DOI: 10.31579/JCRR/2019/001

Copyright: () 2019 Giulio Perrotta. This is an open access article distributed under the Creative Commons Attribution License, which permits unrestricted use, distribution, and reproduction in any medium, provided the original work is properly cited.

Abstract:
The strategic theoretical model is mainly based on the patient's perceptive-reactive system and its
functioning profiles, putting the typical nosographic descriptions of DSM-V in the background - in clinical
practice. This methodological choice is aimed at favouring a more integrated and general approach,
enhancing the particular individual components, typical of the patient, far from an excessively rigid
approachable only to cage the patient in a scheme that does not value all the nuances of his clinical
symptomatology. This model is then integrated with other theories able to fully explain the subjective nature
of reality and the re-elaboration of it in a perceptive key.

\section{Contents of the manuscript:}

1. The reality plan and the perceptive-reactive system of the strategic model. Classification and theoretical profiles

In clinical psychology and psychiatry, the analysis of the real plan is a fundamental operation to identify the psychopathological area of the patient and therefore define the neurotic or psychotic matrix of the disorder. A compromise of the real plan would quickly identify the pathology under examination in the psychotic area, based on the described symptoms. [3]

In dynamic psychology, the "principle of reality" represents one of the central points of the theory of S. Freud, starting from his studies up to the theoretical evolutions of currents inspired by him and his dynamic processes. It is no coincidence that the principle of reality is considered the dominant component in the psychic life of the adult, subsequent and substitute (in the psychic development of the individual) of the reduced pleasure principle, which dominates the psychic life of the infant. If therefore, in the early years, we witness an overwhelming orientation of pleasure, in the following years, we should instead see an ever-increasing orientation linked to reality. The reality principle requires the acceptance of a state of tension in exchange, soon, for greater pleasure or less pain. While the pleasure principle seeks immediate satisfaction of need in a completely irrational way, the reality principle pursues the fulfilment of desire by setting extended goals over time and sublimating the impossible immediate fulfilment in substitute representations. In other words, faced with the impossibility of complete fulfilment, the reality principle acts to adapt the satisfaction of the desire to adverse situations. However, the principle of reality and that of pleasure are not to be considered antithetical; they do not act in opposition to each other. Instead, the former helps to resize the latter, forcing it to take into account the actual conditions of action. The principle of reality does not prohibit the pleasure principle of expressing itself but restores it within certain limits of action. [2]

In Freud's second topology (Es, Io, Super-Io), the author theorized the hypothesis that the principle of reality was attributed to the ego and stemmed from the contrast between the id and external reality. Implementing a process of extreme synthesis, the I organizes and manages environmental stimuli, object relations and is the chief mediator of awareness; if the component of the Self enucleates the person in its totality with respect to the environment, the I (inscribed in the Self) is the structure that perceives itself and enters into relationship with other people (with "their"-I), distinguishing them as "not me". [2]

Freud, the father of the classical psychoanalytic movement, considered the ego as a psychic instance, that is to say, an organizing structure that has the task of mediating social drives and needs, represented by two other conflicting instances (the id and the Super-Ego). The ego manages the defence mechanisms, the psychic processes assigned to the protection of the ego with respect to too intense instinctual experiences or other threatening experiences. Some examples of defence mechanisms are removal, sublimation, reactive formation, splitting, projection. A psychoanalytic school created by Anna Freud is the Psychology of the Ego, which has mainly dealt with describing the defence mechanisms that the Ego disposes to relate to reality. The Es is instead that intrapsychic instance that "represents the voice of nature in the soul of man". The Es, in fact, contains those drives that are erotic (Eros), aggressive and selfdestructive (Thanatos), which are the human way in which instincts have evolved. It is the most archaic intrapsychic instance of our mind, and it is also called the unconscious (unlike the ego, which is partially unconscious but also contains most of the conscious elements). The Es, according to psychoanalytic theory, consists of a large container of instincts that represent the individual's reserve of psychic energy of sexual matrix and stores an enormous quantity of repressed memories (above all infantile): 
J Clinical Research and Reports

for this reason, according to Freud, its activity can be the cause of neuroses, or disorders arising from the conflict between conscious elements (I) and memories that the Super-Ego would not want to recall. Finally, the term Super-ego indicates that intrapsychic instance which, together with the Es and the Ego, composes the structural model of the psychic apparatus and is that which originates from the internalization of codes of conduct, prohibitions, injunctions, schemes of value (good / evil; right / wrong; good / bad; pleasant / unpleasant) that the child implements within the relationship with the couple of parents. The Super-ego consists of a different set of behavioural models, as well as prohibitions and commands, and represents a hypothetical ideal towards which the subject tends with his behaviour. "It is a sort of censor who judges the deeds and desires of man". Through this instance, a mechanism is established, which leads to the shattering of the ego and its subsequent modification, insofar as it derives from it models deriving from the imposition of others. The superego, in fact, springs from the cultural and educational baggage acquired from infancy by parents and later by other possible educators. If, on the one hand, this sphere has a positive function, limiting human desires and impulses; on the other hand, it instead causes a continuous sense of oppression and non-fulfilment. [2]

Still on the subject of the "principle of reality", deviating from the dynamic matrix, the author and researcher Bruner highlight and identifies the difference between two levels of reality:

1) reality understood as an external world;

2) the reality of consciousness as an inner world.

This difference is significant because it helps us to better understand the complexity of the subject under consideration and the two different ways of thinking. One proceeds by sequentiality, helping to create general concepts and categories based on data links by the principle of causality, and is defined logical-paradigmatic and is at the basis of scientific knowledge. The other is the narrative or syntagmatic thought, which proceeds by intention and, unlike the paradigmatic one, does not follow the causal logic but rather that of emotions, beliefs, affections.

Bruner argues that intentionality underlies the construction of reality. In fact, what makes each person different from the other, is the ability to "live" the same event in a different way. The way we organize and signify the experience is different for each of us. The filter through which we interpret reality is the filter of subjectivity, and it is thanks to this that the same event can be read with different modes, filters. Unlike an "objective" vision, reading events through the lens of subjectivity makes every person unique, because the reading that will give of reality, of an event will inevitably pass through its whole system of beliefs, affections and emotions and therefore it will be affected. This differentiates the way of signifying an event from person to person, but also in the same person at different times in his life. The objective of narrative thought is to combine the syntagmatic and narrative plan; therefore, the internal and external world. The narrative, in this perspective, is intended as a device of the mind that builds narratives. The narratives, therefore, do not arise from a simple objective recording of data and events but are the result of an emotional, cognitive and affective re-elaboration that shapes them. [2]

The "strategic model" clearly embraces this line of interpretation and focuses its clinical activity on the theory of the "perceptive-reactive system", that is, the set of redundant modes with which everyone perceives reality, gives it a specific meaning and reacts to it. The underlying assumption of the strategic approach is that there is no real reality in itself, but as many realities as there are different interactions between subject and reality, or how the individual perceives reality and how it relates to itself, with the others and with the world. From this concept, it follows that any condition that man finds himself living is the product of the relationship between himself and what he lives. In the wake of this perspective, the disorder is seen as being produced by rigid and redundant ways of perceiving and reacting to reality, the so-called attempted dysfunctional solutions, that is all that is put in place by the person and/or the system around the person to manage a difficulty and which, instead of leading to the resolution of the problem, maintains and nourishes it, leading to the structuring of a real disturbance. Still in reference to this system, reality is framed in two different sub-types: the "first-rate reality", understood as the objective reality (e.g. the presence of a table in a room); the "second-order reality", understood as the meaning that the person attributes to a given fact (e.g. during a discussion, the contrary thought is interpreted as constructive or as destructive and offensive, based on one's own internal perception scheme). [4-6]

\section{Sensations and perceptions: the "subjective" interpretation of reality}

The distinction between first and second-order realities is of fundamental importance in the understanding of reality and in the communicative processes between living beings, in particular for the pragmatic model of communication developed by the school of Palo Alto. The difference between the two levels mainly concerns the perception of reality which differs from the interpretations we give to it. The perception we have of something material, of an object, even the simplest, can be the same with respect to the people around us at the same time and, they are also observers of the same object in question. The real difference lies in our personal way of communicating around this perception, that is, not objective knowledge, but the meaning that each person personally attributes to the object or situation in question. It is this interpretation that feeds the interaction between several individuals and it is always on these that reality is built. Therefore, there is no single unambiguous version of it, and only if there is a concrete agreement between the interacting subjects on the multiple variations that can characterize it, can communication exist. In the psychological and communicative field, one cannot exempt oneself from the evidence that one always acts on the way of constructing reality with other ways of interpreting it, then on and with second-order realities. [4]

Here it is clear the need to clarify the theoretical and practical difference between "sensation" and "perception". The sensation is clearly the product of stimulation and receptor reactivity, through the five senses (sight, hearing, smell, touch and taste); perception, on the other hand, is the elaboration of the elementary sensation that comes to the sense organ and therefore is the most delicate and sensitive process, since on the basis of the re-elaboration we perceive then the reality around us. [1]

Thanks to the studies of Weber [1] (on the differential threshold or the relevance threshold of the perceived stimulus) and Fechner (on the sensation, directly proportional to the logarithm of the intensity of the stimulus), we have come to say that:

1) the thesis that claimed "sensation = perception" (naive realism) was wrong, while the hypothesis that provided for analytical difference (the so-called critical realism) was correct;

2) only the stimuli to which the sense organ is sensitive are perceived;

3 ) the stimulus, to be recorded by the sense organ, must be sufficiently intense, in an absolute sense.

In the context of "critical realism" then five different and main theoretical approaches were distinguished [1]:

A) the "associationist or atomistic approach" of Helmholtz (1878), which envisaged a perceptive system composed of 2 relatively separate but communicating systems: elementary sensations (I) and cognitive apperceptive layer (II);

B) the "Gestalt approach", which preferred to pay attention to form and representation. Among them:

a) for Metzger, the level of visual perception is not flat but threedimensional; 
J Clinical Research and Reports

b) for Rubin, the visual field is differentiated in background and figure;

c) for Wertheimer, the perceptual field was organized by a set of rules:

- good form (the perceived structure is always the simplest);

- proximity (the elements are grouped according to the distances);

- similarity (tendency to similar group elements according to similarities);

- proximity (tendency to similar group elements according to the close distance);

- closure (tendency to similar group items based on known closed forms);

- continuity (all elements are perceived as belonging to a coherent whole);

- the common destiny (if the elements are in movement, those with a coherent displacement are grouped);

- the figure-background (all the parts can be interpreted both as object and as background);

- pregnancy (if the stimuli are ambiguous, the perception will be reasonable based on the information captured by the retina).

C) the "cognitive approach", which included the integration of the models:

a) "top-down", or the top-down processing (guided by the experience that influences perception);

b) "bottom-up", or the processing from the bottom up (guided by the sensory data elaborated in a cortical way). A typical example is Gibson's ecological approach, which includes very specific assumptions:

- the stimulus is described not in terms of retinal projection but in terms of "optical set-up" (i.e. image that reaches the retina);

- the stimulus is perfect as it is;

- the set of information given by the context and the movement of the observer is called "optical environment".

\section{Among the main perceptive rules we have those concerning:}

1) The "perception of the scene". The scene is an image of the environment and the real world, which contains objects in the foreground and background elements. The degree of perception of a scene is equal to $250 \mathrm{~ms}$ (1/4 of a second), however, Fei has shown that based on the scene (from the least complex to the most complex, taking into consideration the amplitude, the details, the perspective, the colour and the light) we employ (as reaction time) from $25 \mathrm{~ms}$ $(1 / 40 \mathrm{sec}$.$) to 66 \mathrm{~ms}(1 / 15 \mathrm{of} \mathrm{sec}$.).

2) The "movement". The brain perceives movement and processes information. The ability to perceive movement, however, can suffer errors:

a) the induced movement consists in the fact that an immobile object seems to move when a moving object is close (e.g. moon-clouds);

b) the autokinetic effect consists in the fact that if there are no nearby reference points, an object seems to move by itself (e.g. solitary star in the sky);

c) suggestion;

d) hallucinations;

e) the adaptation to the movement consists of that phenomenon whereby if we look at an object in motion fixed, after a specific time we will become less sensitive in perceiving the movement;

f) the apparent movement (phi effect) is that illusion determined by the movement, provided that the frequency of the apparition and disappearance is around 25-30 times per second (e.g. the frames of cinema or television)

3) The "depth". It is the capacity learned during the first year of life (and not innate) and consists of the distance measured along the vertical between the bottom of a hollow body and its upper end. Although we see in two-dimensionality, we are able to perceive three-dimensional space; this assumption is demonstrated by the existence of the following indications:

a) "Physiological Indices":

I) convergence is the ability of the eyes to bring both visual axes of the eyes onto a point of fixation in space;

II) the accommodation is used to focus the images on the retina and is a mechanism dependent on the autonomic nervous system, therefore independent of the will of the subject (it is the brain that establishes the focal point);

III) the binocular disparity is that mechanism according to which the image on the left retina will be slightly different with respect to the one on the right: the entity of the disparity varies with the distance of the object, so the farther it will be, the less the disparity will be;

b) "Pictorial Indices":

IV) the interposition is that mechanism whereby if the image of an object partially covers the image of another object, the first is perceived as closer than the second;

$\mathrm{V})$ perceptive constancy is the tendency of perception to maintain constant characteristics in time and space, within certain limits, even if the stimulation situations are objectively changed. The constants can be of a size, shape, colour (or colour) and clarity:

a) Size: a person $1.70 \mathrm{~m}$ tall, who is at $2 \mathrm{~m}$. or at $10 \mathrm{~m}$., it appears to retain its greatness, despite the fact that the size of its image in the retina changes considerably;

b) Shape: if we observe a disc placed in an inclined position, it will still appear to us as a circle, despite the fact that the image projected on the retina is an ellipse;

c) Colour: if we observe a white object with red illumination, the object is always perceived as white;

d) Clarity: if we observe a piece of black paper in the daylight of a window, we can see that it continually appears black, despite the fact that the amount of light reflected from its surface varies considerably.

VI) shading.

c) "Perspective indexes":

VII) the texture gradient is the mechanism that the mind has to perceive the distance and the depth and it is the surface texture: the more in a given point the single elements appear to us thick, the more we perceive that point as distant;

VIII) the aerial perspective is that mechanism by which an object, whose surface is not perceived with the precision of details, is placed at a greater distance;

IX) the position with respect to the horizon;

$\mathrm{X})$ the linear perspective is that mechanism by which experience makes us perceive objects whose angle of view is smaller (for example, in a path, the last trees compared to the first).

d) "Movement Indices":

XI) the parallax of movement is an effect that occurs every time one moves: the near objects appear to disappear quickly behind us, while the most distant objects seem to move more slowly;

XII) the kinetic effect of depth is an effect: if an observer moves to the side, some objects are hidden by others, while other objects appear that were previously hidden. This allows us to understand which object is in front of us as we move.

4) The "colour". Colour is perceived through a complex series of biochemical and neural events, which transform light (characterized by a given wavelength) into what we call "colour". The wave is characterized by a vision called "tricomatrix" (i.e. based on 3 types of receptors): red/green/ blue; the colour is therefore given by the wavelength of light that hits the retina. A white object is such because it reflects all the wavelengths, while a black one absorbs them all. The 
J Clinical Research and Reports

perception of colour is therefore based on a constructive and subjective process: colour is not a given but appears to us, since it does not exist in nature; in fact, objects have no colour but simply reflect light in a certain way. The formation of colour perception by the eye takes place in 3 distinct phases:

a) in the first phase, a group of photons (visual stimulus) reaches the eye and reaches the photoreceptors of the retina (rods and cones), from which it is absorbed. As a result of absorption, photoreceptors generate (in a process called transduction) 3 nerve signals, which are electrical signals in amplitude modulation;

b) the second phase still takes place at the retinal level and consists of the processing and compression of the 3 nerve signals, and ends with the creation of the opposing signals, frequency-modulated electrical signals and their transmission along the optic nerve;

c) the third phase consists of the interpretation of the conflicting signals by the brain and in the perception of colour.

D) the "constructivist approach" [1], which finds its foundation in the following statement: "perception is an inferential process (i.e. based on the hypothesis), given that the sensory information coming from the external environment is ambiguous and incomplete, to arrive the perception of top-down processes is necessary". Therefore, perception:

a) it is an inferential and active process;

b) is the final process of the interaction between stimulus (from below) and experience (from above);

c) is a process that suffers the influence of various external factors (e.g. vision disorders, psychiatric disorders, incorrect perception, illusion, hallucinations, delusions, suggestion, the impossibility of perception due to the sense organ).

Among the significant exponents we remember:

a) Gregory: starting from Gibson, he states that the starting point is the external stimulus but then we activate the best possible interpretation to explain the complex object),

b) Necker: between two possible interpretations, one will always prevail;

c) Allport: proposed the "perceptive set", or the idea that the field is influenced by motivations, emotions, experiences and subjective expectations, reorganized according to two cognitive operations: the "generalization" and the "categorization".

E) the "synthetic approach" [1], or the schools of thought belonging to the neurosciences, which try to overcome the top-down/bottom-up relationship, integrating, in fact, two models. Among the significant exponents we remember:

a) Bruner: perception depends on one's needs, expectations, moods, subjective values, emotional meaning and personality characteristics (New Look School);

b) Neisser: the brain is a computer and perception is nothing but an analysis by synthesis, the result of a 3-stage sequence:

I) stimulus selection through a pre-attentive automatic process (bottom-up);

II) the voluntary shift of attention to the stimulus (top-down);

III) the final mental representation.

c) Marr: perception can be investigated on at least 3 levels (computational theory):

I) computational level (the end);

II) algorithmic level (the means used);

III) procedural level (the how).

Marr's approach is firmly "bottom-up" (as it focuses on sensory processing) but includes the intervention of "top-down" factors (i.e. information previously learned from the world)":" the chair must have 4 legs to be able to stand in the foot. Thus our knowledge of the world (top-down) acts on the sensory input (bottom-up), reaching a perceptive synthesis $>>$.

The analysis of visual sensory input proceeds for 4 specific stages: a) description of grey levels; b) primitive geometric primitive sketch;

c) sketch with 2 and 1/2 dimensions (e.g. depth);

d) $3 \mathrm{D}$ representation (three dimensions).

However, this model is sharply criticized because it does not take into account an adequate explanation of how the factors at playwork and there are no functional experiments outside the laboratory context.

\section{The defence mechanisms}

Another element that intervenes in the perception of sensory signals, in addition to the perceptive rules and the perceptive-reactive system, is undoubtedly the group of "defense mechanisms" directed by the ego, or those psychological processes, often followed by a behavioral reaction, implemented to deal with difficult situations, to manage conflicts, to preserve their functioning from the interference of disturbing, painful and unacceptable thoughts, feelings and experiences. They are generally (but not necessarily) automatic, as they often work without a conscious effort, as they are a unique tool for dealing with a real or perceived danger. [2]

Over the decades, they have been studied by different authors; among the primary [1]:

1) S. Freud, who considers them first as "unconscious operations for defensive purposes" (c.d. repression), then as "conflict management techniques", identifying four main ones: removal, sublimation, displacement and reactive formation;

2) A. Freud, who after thirty years of clinical and theoretical work, wrote a broad, though not exhaustive, classification of defence mechanisms, known as Hampstead Index;

3) Hartmann, an exponent of ego's psychology, who confirmed the hypothesis that the defences were operations carried out by the ego, using partially neutralized, i.e. depersonalized, aggressive energy;

4) Kernberg who underlined the function of defensive mechanisms, not only aimed at conflict management, but also at the construction and development of the Self, at the representation of objects and at the regulation of object relations.

5) Kohut supports the adaptive role of defences, as they safeguard the integrity of the Self;

6) Vaillant is the father of the modern classification of defence mechanisms, author of one of the most important theoretical and empirical systematizations. He argues that, for an operation to be defensive, it must have been consolidated over time and taken on an adaptive function. The most adaptive defences are those that allow for the gratification of the drives, minimizing the distortion of reality, while the maladaptive ones are used at the price of a significant distortion of reality. Together with Perry, they hierarchize defences into seven distinct levels of maturity and adaptivity. The maladaptive potential of a defence depends on exclusivity, since a specific defence is used repetitively, rigidly and narrowly; by intensity, that is by the quantitative impact of the defence; from age adequacy, as the defences may be more or less functional based on the age of the subject and the life cycle stage; from adequacy to the context. Therefore:

I) the seventh defensive level is highly adaptive and includes defences such as altruism, affiliation, repression, humour, sublimation. These defences promote functional, balanced and socially useful behaviours, allow for gratification and often presuppose an awareness of feelings and their consequences;

II) obsessive defences (6th level - retroactive cancellation, intellectualization, isolation of affection);

III) neurotic defences (5th level - such as removal, dissociation, reactive formation, displacement);

IV) narcissistic defences (4th level - idealization, omnipotence, devaluation); 
J Clinical Research and Reports

V) defences of denial (3rd level - denial, projection, rationalization);

VI) borderline defences (2nd level - projective identification and splitting);

VII) the level of acting out (1st level), also defined as "pulse", which consists in dealing with stress with direct and often impulsive action, implemented without worrying about the consequences. It is the case of a student who, after having taken a bad grade in the interrogation, throws the books with violence;

VIII) There is then another level of defences, the zero level, which indicates a condition of failure of defensive regulation up to a break with reality. It includes psychotic denial, psychotic distortion and delusional projection.

\section{Human needs. [1]}

At the centre of human psychology, there are the "needs", and precisely these inner elements contribute to the subjective analysis of the perceived reality by the subject. In fact, one's needs can influence perceptual analysis, completely altering the sense of reality, encroaching on what is said: "you saw what you wanted to see". On the other hand, it is undeniable that inner needs guide our actions, even in the absence of awareness of their existence or their real consistency.

Maslow devoted his studies precisely to the needs, theorizing the "pyramid". Between 1943 and 1954 he conceived this hierarchy of needs on a pyramidal scale, divided into five levels, from the most elementary (necessary for the survival of the individual) to the more complex (social). The individual is thus realized by passing through the various stages, which must be satisfied progressively. This scale is internationally known as "Maslow's pyramid". The levels of need conceived are:

a) physiological needs (hunger, thirst, etc.);

b) needs for safety, security and protection;

c) membership needs (affection, identification);

d) needs for esteem, prestige, success;

e) needs for self-realization (realizing one's identity and expectations and occupying a satisfactory position in the social group).

Subsequently, they came to criticize this scale of identification, because it would excessively simplify human needs and, above all, their level of importance. The scale would, therefore, be more correct in terms purely functional to the simple survival of the individual than in terms of social affirmation. This would, therefore, be psychophysiological needs, rather than psychological in the strict sense. Other criticisms focused on the fact that the succession of levels may not correspond to an actual state that can be shared by all subjects. Maslow himself in the 1968 book will add some levels he had initially ignored.

Always the author then leaves room for the concept of "motivation", understood as a force that makes us act, according to our needs, or the driving force that seeks gratification and satisfaction, to fill the "needs of lack", or feeling of emptiness and lack that push to search for something, related to primary needs (survival) and "growth needs", or tending to growth, increase motivation and are linked to secondary needs (realization of the person).

\section{Personal constructs and the belief system [1]}

To the perceptive rules and to the perceptive-reactive system, beyond the defence mechanisms and human needs, we add the "personal constructs" that each of us possesses, as the result of subjective re-elaborations of reality.
Kelly argued that reality, even if it contains objective elements, is always a subjective reworking, according to eleven corollaries identified by him, among which the following maxims stand out:

a) the event is anticipated by subjective psychological perception;

b) the interpretations of the events are subjective;

c) thought is inherently dichotomous (good-bad, right-wrong);

d) every action is the result of a subjective choice;

e) subjective experience moves choices;

f) behavioural changes are possible provided that modulation is the result of a subjective choice.

The world view is thus generated through the use of "constructs", i.e. units of knowledge through which people structure their own reality, give meaning and meaning to the world, organizing evaluation and anticipation criteria; they are personal, they have a bipolar structure and their meaning does not correspond to those suggested by ordinary logic. The organization of constructs can give shape to dysfunctional realities for the person himself since every form of change involves modification and a repositioning. The critical points of his theory (of personal constructs) are therefore the following:

1) the processes of a person are psychically channelled by the way in which it anticipates events;

2) the person is not moved by chance but acts according to the "trajectories", or modalities of anticipation of events, characterized by flexibility, subjectivity and awareness;

3) human activities are guided by "constructs", or modalities of knowledge of reality;

4) the "system of constructs" is an organized representation of reality but on a personal (subjective) level, where knowledge is organized and structured to interpret reality and minimize incongruent or incompatible elements. It follows an ordinal hierarchy and contains "core" constructs (which define identity) and "peripheral" (subordinate) constructs.

Our evaluations and our thoughts therefore often derive from deeply rooted "beliefs", which concern ourselves, the world and others, and which are able to influence emotions and behaviours significantly. According to Ellis, it is possible to identify and modify all those thoughts that generate maladaptive emotions and behaviours, thus helping the person to be less vulnerable to emotional disorders and maladaptive behaviours. Here is a list of the main dysfunctional thoughts, from which many others derive:

1) We must always be loved or appreciated by all significant people. I must always have the approval and love of all the people I consider important or significant.

2) You must always be right and competent to be considered worthy of value. I have to prove myself always competent and adequate in everything I do.

3) Some people are completely cynical, evil and deserve to be severely condemned and punished. If the others mistreat me, I must be severely punished, and I have to do so that sooner or later, I get it.

4) It's terrible and catastrophic if things don't go as we want. Things have to go the way I want because I absolutely need to have what I want, and if I don't get it, then my life is horrible.

5) Human suffering depends only on external causes, and we can do nothing to control or change our emotions. If something unpleasant or harmful happens to me, I can do absolutely nothing to improve the situation, so I have to suffer forever, and then my life is no longer worth living.

6) If something is or can be dangerous, you have to worry about it a lot and think about it all the time.

7) It is better to avoid specific difficulties than to face them.

8) You must necessarily depend on others and have someone stronger to rely on. I consider myself weak and inadequate, so I need to depend on others. 
J Clinical Research and Reports

9) What happened to us in the past will continue to influence our lives forever. My parents should have treated me differently as a child, and my troubles now depend on my past.

10) We must much upset ourselves about the problems and disturbances of others.

11) There must always be a right and perfect solution to any problem, and it is a horrible thing not to be able to find it. I must find perfect solutions to my problems and those of others. Otherwise, catastrophes and horrors will happen.

12) I must not feel anxiety, depression, guilt, shame, anger, and if it happens to me, then I am a weak person and to be condemned.

13) My unhappiness depends on others, and I must continue to remain anxious and depressed if others do not change.

These ways of thinking concern the rules that each of us gives and the relationships we have with others, with the outside world or with ourselves, and can be traced back to the following necessary attitudes:

a) "absolutization" which is expressed in total and absolute judgments on others and on oneself, absolute needs and indispensability, absolute unbearably and emotional intolerance, catastrophism;

b) "indebtedness" (I must, you must, ...) on oneself and on others.

From these "dysfunctional thoughts or irrational ideas" derive the "automatic thoughts" that condition our reactions on an emotional, behavioural and physiological level. The automatic thoughts are cognitive distortions that appear without us fully realizing it:

a) "dichotomous thinking": all or nothing (often related to absolute judgments), there are no intermediate degrees, if a situation is not perfect it is a complete failure;

b) "hypergeneralization": doing, as they say, "a whole bunch", often related to duties and absolute judgments;

c) "labelling": defining things and people with a global label instead of referring to specific elements;

d) "mental filter or disqualification of the positive" (often related to catastrophism);

e) "hasty conclusion" (often related to judgments or absolute needs): arriving at a judgment or attributing meaning in a hasty and superficial way;

f) "emotional reasoning" (often associated with unbearable and absolute needs);

g) "must-should" (related to absolute duties);

h) "personalization": holding oneself responsible for something which, in reality, other people or other factors are responsible for (often related to unbearable or absolute needs).

If our evaluations and attributions of meaning are affected by these expectations and beliefs, we will have stress and unpleasant emotions assured. Dysfunctional thoughts distort reality. They do not help to overcome difficulties and lead to unpleasant emotions, sometimes particularly intense. Useful thoughts do not magnify facts or devalue things and people. They help overcome difficulties and allow you to experience an adequate state of mind to deal with life situations.

The "Paradigm $A-B-C-D-E$ " describing the process examined can help us defuse our dysfunctional thoughts and have functional effects too. Activating Event;

b. Dysfunctional thoughts;

c. Emotional, behavioural and physiological consequences;

d. Questioning of B, restructuring;

e. Effects of questioning: effect on emotions and behaviour.

However, free will [7] is, from a neuroscientific point of view, a mythological animal still to be studied. Free will is associated with the ability to make choices, but, obviously, the possible choices are limited to the options that are available, therefore limited by the physical state, by previous personal experiences, by the cultural context and, mainly, by the emotional state. There are therefore factors, not entirely impeding, but positively influencing free wills, such as for example emotions, social pressure, conventions, moral norms, convictions, prejudices and superstitions. Furthermore, free will may be related to other bio-psychosocial factors such as genetic predisposition, the expectation of reward, avoidance of risk, fear of punishment. In particular:

a) "Reward" is able to influence behaviours and therefore limit free will, in the sense of favouring the choice of the best option that guarantees survival. The brain circuit of reward includes various areas and nuclei (of which the main one is the nucleus accumbens) which mainly works with the neurotransmitter dopamine (DA). Dopaminergic stimulation influences the choices and the reaction times and, therefore, enters the free will mechanism: a strong reward signal in dopaminergic neurons exert an action on the behavioural objectives that can infer on the choice of an individual. When dopamine is excessively increased, for example pharmacologically with prodopaminergic drugs (one of which is pramipexole, used in Parkinson's patients), pathological gambling behaviours can be generated, in which the exasperated search for winnings is a strategy which leaves no room for deliberate choices of behaviour. There are many psychophysical dimensions that are able to interfere with reward mechanisms and, therefore, with free will. Among the main ones, it is possible to remember impulsiveness, compulsion, some personality traits, anxiety and stress, mood depression, especially in its anhedonic component and addictive behaviours.

b) "Risk avoidance" is a temperament that is frequently present in patients with obsessive-compulsive disorder (OCD), with the aim of avoiding potential losses. However, in these subjects more recent neuropsychological and neuroimaging data have found an increased impulsivity, with decisional aspects at risk and a dysfunction of the reward circuits, assimilating in this sense those subjects to individuals with behavioral dependence, as the reduction of the stress, resulting from the implementation of compulsive behaviour, acts as a reward in itself. Also, in this situation, the reduction of free will is visible. In other words, the anxiety induced by obsession (cortical circuits) and the consequent need to satisfy the obsession by implementing a compulsion (subcortical circuits), sharply limits the scope of free choice.

c) "Fear of punishment" is a significant factor, after that of reward mechanisms, in the emotional limitation of a free will. The brain circuits that guide decision-making capacity within a fear of punishment are complex (amygdala, posterior cingulate cortex, right dorsolateral prefrontal cortex) and move within a balance between an assignment of appropriate punishment (emotional control) and a correct determination of the levels of responsibility (executive control). The stimulation of the brain circuits regulating the experience of punishment can inhibit the gratification circuits. The subject, in an attempt to find escape options from the presumed punishment, suffers a significant limitation in the free choice, putting in place a limited deliberative ability and scarce proactive behaviours.

\section{The social categories and the influence of attitudes and reputation [1]}

"Social cognition" is the way in which people come to consider, in addition to knowing, their social world. In particular, there are three essential elements:

1) "Attitudes" are the evaluative representations of the social world by men. The first 2 to talk about it were the sociologists Thomas and Znaniecki, starting from the '20s from the idea of seeing in the attitudes of the antecedents of behaviour; subsequently, Rosenberg and Hovland spoke of a "tripartite model", that is the attitude as a psychological construct made up of three components: cognitive, affective and behavioural. There are 3 ways of forming attitudes: direct experience, the experience of others and communication. However, however, the attitude is not always an indicator and predictive of behaviour: in fact, for Fishbein and Aizen (theory of 
J Clinical Research and Reports

reasoned action), the attitude and situational and contingent factors lead to behaviour.

2) "Impressions" are the sensations that make us move effectively in the social world and understand reality. What is known is that the formation of an impression is a sequential model given by the acquisition and progressive accumulation of information. In forming an impression, we actively search for information that could be useful for our goals: we can proceed either by seeking confirmation of our initial hypotheses or by looking for empirical evidence that contradicts them. However, not infrequently, the error appears, perhaps due to overestimation or due to conditioning or to voluntary illusions (which lead us to believe in the tendency to believe that two events are associated with one another even when in reality such an association is not present).

3) "Reputation" is a judgment formulated by a community about an individual who generally belongs to the community itself. Reputation on human qualities that are difficult to observe and on relatively rare characteristics. The purpose of reputation is to ensure cooperative exchanges and behavioural expectations of others (Emler). Showing a positive personal reputation gives good credit and promotes selfcontrol, while if it is negative, it becomes a form of correct labelling by the community that makes it a vicious circle.

Perception consists of an active process of constructing reality, starting from external stimuli; social perception is the "way in which we subjectively create a series of impressions on others and make judgments about others, even on the basis of inferences". The way to deduce impressions and judgments on others is based on a necessary organization of data which, in the case of social perception, concern, for example, the characteristics of the other person such as age, sex, gestures, non-verbal communication etc.

Our social perception can also be constructed through processes of causal attribution: according to the theory of causal attribution (Heider, Jones and Davis), we come to produce social judgments by attributing a causal relationship between the actions of others and particular causes or events: in in other words, when we analyze the behaviour of others we usually use explanations that are attributable to the personal characteristics of the people involved or to the characteristics of the situation.

There are two relatively opposite approaches to explain the perceptive processes that direct our social judgments, both oriented towards going beyond the given:

a) the "bottom-up" approach restricts the formation of social perception to the information provided by the environment (e.g. Mario often talks to the elderly at the park $\rightarrow$ Mario is a sensitive person);

b) the "top-down" approach maintains that we actively organize the surrounding world, using a deductive process starting from already existing constructs (e.g. Mario is a sensitive person $\rightarrow$ Mario would like to talk willingly with the elderly).

Asch showed that the perception of another person is not the simple sum of concepts or ideas used to describe it, in other words: "the whole is much more than the sum of the single parts." primacy effect:

1) the "social perceiver" tends to attribute a small nucleus of central aspects to the person in front of him, to create his impressions on it. This was derived from his experiment: $\ll$ Asch gave 2 groups of subjects a list of same adjectives that described a person, with the only difference that in a group the list began with adjectives with a negative value (e.g. superficial) and in the 'other group with positive adjectives (e.g. kind). Asch observed that, although the other adjectives in the list were the same, there was an offset between the 2 groups in the way they perceived the person: he concluded that the first traits of the list are more influential because they are the ones that activate the global configuration of the impression. In essence, the first impression is what counts $>>$;
2) the need to restrict information to "mental categories" derives from the need to reduce the quantity of inconsistent or ambiguous information to ensure a complete understanding of the corporate purpose.

\section{Systematic errors: impressions, beliefs, cognitive dissonances and moral disengagement [1]}

On the basis of one's beliefs, one's system of personal constructs and social categories, the defence mechanisms of the ego and one's own inner needs, the person has his own and subjective perceptive-reactive system, with which he interprets reality. This operating procedure, however, precisely because it is subjective, is subjected to evaluations and judgments that may not be consistent and compatible with the "subjective" reality of the other person, thus falling into "systematic errors" in the interpretation of reality.

First of all, as we have already seen, the "impressions" can negatively affect the correct interpretation. These mental representations are used to obtain a presumed coherent and stable knowledge structure of the other, starting from known elements (e.g. environmental context, previous information, physical aspect, non-verbal communication, manifest behaviour). Impressions are different; however, from sensations, since the latter is the result of an external stimulus not yet transformed into perception.

In secundis, we find "effective heuristics", understood as cognitive strategies, mental shortcuts or in any case non-rigorous and hasty procedures that rely on intuition and subjective perceptions. "Effective" because despite the lack of rigour in the procedure, the result is still positive and satisfying (e.g. my instinct was right, you are a terrible person ...). Among the most famous, we remember:

1) "availability", when we judge the probability of an event based on the ease of imagining examples, or on the mental availability we have of them: if the examples come to our mind quickly we conclude that the probability of that type of event is high;

2) "simulation" (or counterfactual reasoning), when one imagines how different results could have been verified from those that actually occurred if that event had not occurred;

3) "of the anchoring and adjustment", when we are influenced by the previous or expressed opinion of the speaker first, it exerts a considerable influence and the subsequent judgments never move much from it;

4) "of representativeness", when it allows us to reduce the solution of an inferential problem to a particularly simple judgment operation. This heuristic induces to evaluate the probability of a hypothesis, based on a similarity judgment or consists in the classification of a case based on the similarity with the typical case.

In terzis, we find "cognitive distortions" (or "prejudices" or "cognitive bias"), understood as constructs founded, outside of critical judgment, on wrong or deformed perceptions, on prejudices and ideologies; often used to make decisions quickly and effortlessly. In summary: if the heuristics are convenient and rapid shortcuts extrapolated from reality that lead to fast conclusions, the cognitive biases are cognitive distortions, or ineffective heuristics, abstract prejudices that are not generated on real data, but are acquired a priori without criticism or judgement. Among the best known:

1) "confirmation", that is the prejudice that drives us to surround ourselves with people who give us a reason and who confirm our point of view;

2) "in-group bias", that is the prejudice that pushes us to overestimate the skills and value of our group;

3) "anchor" (or relativity trap), or the prejudice that drives us not to consider any positions that we do not like or do not convince us, anchoring us to the idea of departure;

4) "projection", that is the prejudice that leads us to believe that the majority thinks like us; 
J Clinical Research and Reports

5) "negativity", that is the prejudice that leads us to evaluate harmful elements as most important;

6) "of change" (or of the status quo), or the prejudice that drives us to fear change and to resist it;

7) "del peacock", or the prejudice that drives us to share our successes more than our failures;

8) "the illusion of frequency", or the prejudice that leads us to overestimate the frequency of information that concerns us;

9) "of the present", that is the prejudice that pushes us to want everything and immediately, making decisions to obtain immediate gratification, ignoring the possibilities of gain deferred over time;

10) "optimism", that is the prejudice that drives us to be more optimistic than realists;

11) "of omission", that is the prejudice that leads us to prefer choices that involve omission rather than action, even when this means exposing oneself to objectively high risks;

12) "action", or the prejudice that leads us to strive for action even when reflection would have been the best solution;

13) "probability": we know several:

- "Gabler fallacy", that is the tendency to give relevance to what happened in the past, so that the current judgments are entirely influenced by these past events (for example "if black is released four times, then it is more likely to come out red");

- "greater confidence", that is, individuals, express increased confidence in their abilities that is not justified by real data (e.g. considered to be better than others in guessing numbers, in understanding the mechanisms underlying the game, in the implementation of effective gaming strategies);

- "tendency of the numbers", that is to say, tendencies and "laws" are identified in relation to random distributions (typical error of those who think that the "latecomers" numbers are more likely to be extracted, or that a number just drawn is not probable in subsequent drawings) );

- "illusory correlations", that is when two events belonging to different domains of reality are judged to be interdependent if they occur concurrently (e.g. buying a lucky scratch card and continuing to go to the same bar to buy more). It is also the basis of ritual-stamic and superstitious behaviour;

- "winnings from others", or a logical error that distorts the estimation of probabilities in a rather direct manner and provides the conviction (belief) that "winning" is an event that happens regularly and that "to win just keep playing". The illusions can be of 2 types: primary, if you make faith in calculations and mathematical techniques; secondary, if we believe in superstition.

In quartis, we find the "cognitive dissonances", theorized by Festinger in the framework of consonances/dissonances. In essence, this concept is used to describe the situation of complex cognitive processing in which beliefs, notions, opinions expressed simultaneously in the subject in relation to a theme are in functional contrast between them; examples are the "dissonance due to logical incoherence", the dissonance with the tendencies of past behaviour, the dissonance relative to the environment with which the individual finds himself interacting (dissonance for cultural customs). An individual who activates coherent ideas or behaviours finds himself in a satisfactory emotional situation (cognitive consonance); on the contrary, we will find ourselves in discriminatory and elaborative difficulty if the two representations are mutually opposite or divergent. This inconsistency is the one that produces, in fact, a cognitive dissonance, which the individual automatically tries to eliminate or reduce due to the marked psychological distress that it entails (for example, reduction of self-esteem); this can lead to the activation of various processing processes, which make it possible to compensate for dissonance (and restore self-esteem). An exemplary application of such processes can be had, for example, when a subject who explicitly despises the thieves is found to buy an object at too low a price in order not to sense the illicit provenance. To reduce this contradiction, according to
Festinger, the same individual can, for example, stop despising thieves (thus changing the attitude), or refuse to buy the proposed object (thus changing the behaviour). A famous example of cognitive dissonance is represented in the famous story The fox and the grapes, taken from Aesop's Fables, in which the dissonance between the desire of the grapes and the inability to reach them leads the fox to elaborate the conclusion not supported but from real data - that "grapes are unripe". Summing up, therefore, this concept refers to the quality and intensity of the relationship between two cognitions (i.e. knowledge, beliefs and values), so there is no overlap between two cognitions (with opposite directions). The dissonance (an opposite phenomenon to the consonance) causes a feeling of discomfort that the subject tries to reduce/eliminate:

1) "modification of the cognition responsible for the inconvenience": e.g. A states that work comes first but at the same time states that he must learn to enjoy life. The dissonance between the two hypotheses is evident.

2) "introduction of new knowledgeable about reducing dissonance": e.g. my biggest dream is to become a doctor, but if I do not pass the university admission test, I will do another faculty.

3) "ab abduction reduction the present dissonance": e.g. the wolf that does not reach the juicy grape says it is unripe.

Even Allport, as an exponent of the cognitive approach, gave his contribution, studying the "representation of the real"; according to him, it was based on two different cognitive schemes: the "categorization" and the "generalization". Through the first, the human being selects and organizes the different realities that surround him; with the second, instead, the man tends to attribute to events of a general type the meanings deriving from the few observations made on the available events. These two schemes, again, according to the author, highlight the functional limitations of the human mind, and product evaluations of the wrong type (bias) that can sometimes lead to the labelling of individuals.

In the field of behaviourism, moral development has been studied as an aspect of learning. The individual learns the norms of moral behaviour through a series of experiences in which some acts are subject to positive reinforcements, such as affection, while others to punishments, for example, physical. The behaviourist orientation that has most successfully dealt with moral development is the conception of social learning. In this current of thought, it is considered unlikely that the various morally relevant behaviours are acquired initially by reinforcement, arguing instead that behaviour, to be reinforced, must first occur spontaneously.

The cognitive-evolutionary theories were questioned by Bandura, according to his social-cognitive formulation. The explanatory principle underlying the aforementioned theory is triadic determinism, stating that the functioning of a person derives from the interaction between three factors: the physical and social environment, cognitive and affective systems and individual behavior. Bandura has also investigated the mechanisms and conditions that, in the course of socialization, determine the activation or not of internal moral controls, thus acting as causes of the immoral behavior of people while capable of the highest forms of moral reasoning. Thus it identifies eight main and different mechanisms of "moral disengagement":

1) "Moral justification": the behaviours considered reprehensible are considered from a different point of view and attributable to higher ideals for which they are accepted and justified in their name. Religion, homeland, family and honour are often referred to as excuses for malevolent action;

2) "Euphemistic labelling": attenuation of the violence associated with the words with which it is mentioned, is an example of such expressions as "smart bombs", "ethnic cleansing";

3) "Advantageous comparison": the comparison of behaviour with others in our opinion analogous, leads to the modification of our judgment, attenuating the negative value of behaviours and even transform them into moral actions. "So much more outrageous is the work compared, so much more probable that our deplorable behaviour appears 
J Clinical Research and Reports

irrelevant or even benevolent" stresses Bandura. We must not go too far in carrying out an example of this mechanism: in politics, government officials often justify some incorrect decisions taken against citizens, attributing their responsibilities to the previous government, which would have adopted worse measures;

4) "Displacement of responsibilities": when the active and conscious role of the subject in action is not recognized, his responsibility and consequently the blame also diminish. The responsibility for the action pursued is therefore attributed to higher authorities justifying themselves in an "order" by the latter;

5) "Dissemination of responsibility": from the principle "if everyone is responsible then no one is responsible" shows how moral control weakens when the ability to act and the negative consequences on the victim are masked by sharing the action with others. The Nazi government relied on this type of mechanism by involving the German people to justify themselves to external eyes and to create a justification for the citizens themselves, who thus contributed to operating in the machine of Nazi destruction (in contribution with the displacement mechanism of responsibilities);

6) "No consideration (or distortion) of the consequences": by avoiding thinking about the negative consequences of one's actions, individuals more easily pursue the personal goal setting. This distortion helps to form a distance between the injuring subject and the injured victim, attenuating moral control;

7) "Dehumanization of the victim": this mechanism acts on the empathic capacity of an individual. When a person is "degraded" by his status as a human being, the empathic correspondence of a second subject is lost;
8) "Attribution of guilt": a useful expedient to find a justification for one's own action is to take it out on opponents or with the circumstances that according to the individual would have been provocative and would have triggered the chain of violence first.

\section{Conclusions}

The following conclusion appears evident after examining the question: on the basis of educational systems and cultural contexts, beliefs, conditionings, moral and social norms, systematic errors, neurobiogenetic tendencies and psychological processes such as waiting for reward, avoidance of risk and the fear of punishment, we interpret reality in a subjective way, building our personal way of seeing what surrounds us, based on our perceptive-reactive system, protected by our defense mechanisms and driven by emotional needs and expectations.

\section{References}

1. Perrotta G., Psicologia generale, Luxco Ed., 1th ed., 2019.

2. Perrotta G., Psicologia dinamica, Luxco Ed., 1th ed., 2019.

3. Perrotta G., Psicologia clinica, Luxco Ed., 1th ed., 2019.

4. Watzlawick P., Nardone G., Terapia strategica breve, Raffaello Cortina Ed., 1997.

5. Nardone G., L'arte del cambiamento, Tea, 2015.

6. Nardone G., Il dialogo strategico, Ponte delle Grazie, 2018.

7. Torta R., Il libero arbitrio e le neuroscienze. L'inquadramento scientifico. Bioetica N. Torino, Anno VI, n. 6, 2017. 